\title{
Siamo tutti responsabili per o di qualcosa
}

\author{
Ilaria Bonomi
}

PUBBLICATO: 10 MARZO 2020

\section{Quesito:}

Quale sia la forma da usarsi tra responsabile di e responsabile per costituisce l'oggetto di diversi quesiti sottopostici da lettori. Tra questi, meritano particolare rilievo i quesiti di due lettori, V. A. da Bolzano e R. D. da Vimercate, che oltre a chiedere se sia più corretto responsabile di o responsabile per, notano che in alcuni casi l'uso di per viene più naturale: p.es. "essere responsabile per se stesso" "mi sento responsabile per quanto è accaduto", o anche ipotizzano che ci sia differenza tra "essere responsabili di qualcuno" e "essere responsabili per qualcosa".

\section{Siamo tutti responsabili per o di qualcosa}

$\mathrm{L}$ a lingua italiana, come si sa, presenta un quadro notevolmente complesso e variato delle cosiddette reggenze, cioè dei collegamenti che le preposizioni hanno con i verbi e con i sostantivi e gli aggettivi, con molti obblighi ma anche molte possibilità di variare, a seconda del contesto, nell'abbinare una o un'altra preposizione a un verbo, sostantivo o aggettivo.

Vediamo quali indicazioni emergono dai dizionari e dalla documentazione sull'uso dei nostri giorni offerta da Google libri (9/12/2019).

I dizionari dell'uso registrano in modo univoco la reggenza con di, senza mai indicare la possibilità di costruire l'aggettivo con per (responsabile di un'azione, di un servizio, ecc.).

La documentazione offerta da Google libri è interessante, e si presterebbe a qualche approfondimento di ricerca sulle modalità di alternanza delle due preposizioni. Limitiamoci a considerarne brevemente le cifre, con qualche caso significativo.

"Responsabile di" ha 259.00o occorrenze ( $r$. di servizio, di progetto, di procedimento...), a cui vanno sommate ovviamente quelle con la preposizione articolata: p.es. "responsabile del" ricorre 273.000 volte, "responsabile dei" 79.900, "responsabile della" 253.000, ecc.

"Responsabile per" ha 75.80o occorrenze, con molti esempi di uso di per del tutto equivalente a di: "responsabile per la trasparenza" "r. per l'edizione critica", "r. per l'attuazione", "r. per i danni da lesioni personali", "r. per gli errori o le omissioni".

Considerando che "responsabile per" può introdurre tutta la gamma morfologica del nome (maschile, femminile, singolare, plurale, preceduti dai diversi articoli: per il, per $i$, per la, ecc.), diversamente da "responsabile di", appare schiacciante, nell'uso, la seconda delle due preposizioni, l'unica, del resto, registrata dai dizionari.

Resta la possibilità per il parlante o lo scrivente di scegliere l'una o l'altra senza incorrere in una scorrettezza, ma pensiamo che sia utile questa indicazione: responsabile per è più adatto quando introduca qualcosa di occasionale o temporaneo, non stabile: per esempio appunto "mi sento responsabile per quanto è accaduto" (si veda il quesito di V. A.), che comunque potrebbe avere anche 
la preposizione di, rispetto a un caso come "è nominato responsabile della transizione al digitale della sua azienda". Anche se lo stesso ente istituzionale Agenzia per l'Italia digitale denomina la figura istituzionale sia "responsabile per la transizione al digitale" sia "responsabile della transizione al digitale"! Libertà della lingua...

\section{Cita come:}

Ilaria Bonomi, Siamo tuttiresponsabili per o di qualcosa, "Italiano digitale", 2019, XII, 2020/1 (gennaio-marzo)

DOI: $10.35948 / 2532-9006 / 2020.3270$

Copyright 2019 Accademia della Crusca

Pubblicato con licenza creative commons CC BY-NC-ND 